\title{
Prevalência do diagnóstico de enfermagem Mobilidade Física Prejudicada em pessoas com esclerose múltipla
}

\author{
Prevalence of nursing diagnosis Impaired Physical Mobility in people with multiple sclerosis \\ Prevalencia del diagnóstico de enfermería Movilidad Física Deteriorada en personas con esclerosis múltiple
}

\author{
Thainá Câmara da Silva', Harlon França de Menezes", Rebecca Stefany da Costa Santos'II, \\ Mariana Melo da Cruz Domingos Góis ${ }^{\prime v}$, Olga Alice Alencar Moreirav , Richardson Augusto Rosendo da Silvavl
}

\begin{abstract}
RESUMO
Objetivo: identificar a prevalência do diagnóstico de enfermagem Mobilidade Física Prejudicada, suas características definidoras e fatores relacionados em pessoas com Esclerose múltipla e verificar a associação entre ambos e suas razões de prevalência. Métodos: estudo transversal com 113 pacientes em um hospital da região Nordeste do Brasil. Para a análise dos dados utilizou-se os testes de qui-quadrado de Pearson e exato de Fisher, sendo calculadas também as razões de prevalência. Resultados: o diagnóstico esteve presente em $89 \%$ da amostra e as características e fatores que apresentaram associação foram: alterações na marcha; instabilidade postural; movimentos descoordenados; redução nas habilidades motoras finas e grossas; depressão; força muscular diminuída e prejuízo músculo esquelético. Conclusão: o diagnóstico apresentou-se com elevada frequência na amostra, o que permite identificar as necessidades de intervenções que diz respeito à capacidade funcional do indivíduo, potencializando seu rendimento funcional e pessoal, através do planejamento do cuidado e da assistência qualificada.

Descritores: Esclerose múltipla; diagnóstico de enfermagem; processo de enfermagem, terminologia padronizada de enfermagem.
\end{abstract}

\begin{abstract}
Objective: to identify the prevalence of the nursing diagnosis Impaired Physical Mobility, its defining characteristics and related factors in people with multiple sclerosis, and to verify the association between both and their prevalence ratios. Methods: cross-sectional study, with 113 patients in a hospital in the Northeast region of Brazil. Pearson's chi-square test and Fisher's exact test were used to perform data analysis, and the prevalence ratios were calculated. Results: the diagnosis was present in $89 \%$ of the sample and the characteristics and factors that presented association were: changes in gait; postural instability; uncoordinated movements; reduction in fine and gross motor skills; depression; decreased muscle strength and skeletal muscle injury. Conclusion: the diagnosis was presented with high frequency in the sample, which allows identifying the needs of interventions that relate to the functional capacity of the individual, enhancing their functional and personal income through the planning of care and qualified care.
\end{abstract}

Descriptors: Multiple sclerosis; nursing diagnosis; nursing process; standardized nursing terminology.

\section{RESUMEN}

Objetivo: identificar la prevalencia del diagnóstico de enfermería Movilidad Física Deteriorada, sus características definitorias y factores relacionados en personas con esclerosis múltiple, y verificar la asociación entre ambos y sus razones de prevalencia. Métodos: estudio transversal, con 113 pacientes en un hospital de la región noreste de Brasil. La prueba de chi-cuadrado de Pearson y la prueba exacta de Fisher se usaron para realizar el análisis de datos, y se calcularon las razones de prevalencia. Resultados: el diagnóstico estuvo presente en el $89 \%$ de la muestra y las características y factores que presentaron asociación fueron: cambios en la marcha; inestabilidad postural; movimientos descoordinados; reducción de las habilidades motoras fina y gruesa; depresión; disminución de la fuerza muscular y lesión del esqueleto muscular. Conclusión: el diagnóstico se presentó con alta frecuencia en la muestra, lo que permite identificar las necesidades de las intervenciones que se relacionan con la capacidad funcional del individuo, mejorando sus ingresos funcionales y personales a través de la planificación de la atención y la atención calificada.

Descriptores: Esclerosis múltiple; diagnóstico de enfermería; proceso de enfermería; terminología normalizada de enfermería.

\section{INTRODUÇÃO}

A Esclerose Múltipla (EM) é uma doença autoimune, inflamatória, desmielinizante, crônica que afeta o Sistema Nervoso Central (SNC), mais comum em adultos jovens. A sua etiologia não é bem definida ainda, porém algumas evidências sugerem que esteja relacionada tanto a fatores genéticos como ambientais ${ }^{1}$.

'Enfermeira. Universidade Federal do Rio Grande do Norte. Natal, Brasil. E-mail: thainacamara18@gmail.com

"Enfermeiro. Aluno do Curso de Doutorado. Escola de Enfermagem Aurora de Afonso Costa, Universidade Federal Fluminense. Niterói, Brasil. E-mail: harlonmenezes@hotmail.com

I'Enfermeira. Universidade Federal do Rio Grande do Norte. Natal, Brasil. E-mail: rebecca.stefany@hotmail.com

IVEnfermeira. Universidade Federal do Rio Grande do Norte. Natal, Brasil. E-mail: marimelogois@gmail.com

vEnfermeira. Universidade Federal do Rio Grande do Norte. Natal, Brasil. E-mail: olguinhamoreira@hotmail.com

viEnfermeiro. Doutor. Professor Associado. Departamento de Enfermagem, Universidade Federal do Rio Grande do Norte. Natal, Brasil. E-mail: rirosendo@hotmail.com 
Suas manifestações clínicas iniciam, na maior parte das vezes, por surtos ou ataques agudos. Os sintomas mais comuns são neurite óptica, paresia, ou parestesia de membros (refere-se às sensações cutâneas como formigamento, pressão, frio ou queimação), disfunções da coordenação e equilíbrio, mielites, disfunções esfincterianas e disfunções cognitivo-comportamentais, de forma isolada ou em combinação ${ }^{2-5}$.

A EM afeta mais o sexo feminino, sendo sua razão ainda desconhecida. Porém, as observações existentes apontam que as mulheres são mais susceptíveis as doenças autoimunes, sendo esta predisposição estar relacionada a fatores hormonais ${ }^{6}$.

A idade média da EM é aproximadamente aos 30 anos, com 70\% dos doentes apresentando sintomas entre os 20 e os 40 anos. A idade de início é cerca de 5 anos mais cedo nas mulheres que nos homens. A forma surto-remissão tende a ter um início mais precoce, em média entre os 25 e os $29 \operatorname{anos}^{5-7}$.

A incidência e a prevalência da EM variam em termos de distribuição geográfica. As maiores incidências tendem a ser nos extremos da latitude em ambos os hemisférios. Esta variação geográfica pode ser explicada em parte por diferenças raciais; as populações brancas, em especial as do norte da Europa, são as mais susceptíveis. Outra explicação possível para a associação da EM e da latitude é a exposição solar, que pode ser protetora, tanto pelo efeito da radiação ultravioleta como pela vitamina D. Esta hipótese é apoiada por estudos que encontraram uma relação inversa entre a luz solar e/ou exposição a radiação ultravioleta e a prevalência de EM ${ }^{5-8}$.

No Brasil, embora a distribuição de casos da EM ainda não seja bem conhecida, estudos sobre sua manifestação nos municípios de São Paulo e de Santos revelaram taxas de 15 casos/100.000 habitantes ${ }^{9,10}$.

Segundo o Ministério da Saúde, estima-se que aproximadamente 35 mil pessoas convivem com a doença, sendo que aproximadamente 15 mil estão em tratamento atualmente no Sistema Único de Saúde. Entre os principais sintomas estão fadiga, formigamento ou queimação nos membros, visão embaçada, dupla ou perda da visão, tontura, rigidez muscular e problemas de cognição ${ }^{11}$, sendo necessário acompanhamento profissional, incluindo a enfermagem.

O processo de enfermagem é caracterizado como uma prática sistemática da assistência, cujo objetivo é organizar e planejar a assistência de enfermagem prestada, a partir da identificação das necessidades prioritárias. Os diagnósticos, os resultados e intervenções fazem parte dos elementos essenciais da prática do enfermeiro ${ }^{12}$.

De acordo com a Taxonomia II da North American Nursing Diagnosis Association (NANDA)-I o diagnóstico de Mobilidade Física Prejudicada está inserido no domínio 4, Atividade/Repouso, e na Classe 2, Atividade/Exercício. É definido como limitação do movimento físico independente e voluntário do corpo ou de uma ou mais extremidades ${ }^{13}$.

Destaca-se que a ocorrência do Diagnóstico de Enfermagem (DE) em questão pode provocar o desenvolvimento da intolerância à atividade física, desconforto nos esforços físicos e a fraqueza generalizada, apresentando déficit no autocuidado decorrente do prejuízo neuromuscular ${ }^{14}$.

Daí advém à necessidade de se identificar precocemente o diagnóstico de enfermagem Mobilidade Física Prejudicada para que soluções sejam buscadas pelo enfermeiro junto ao paciente e sua família. Além disso, faz-se necessário, para uma boa atuação do enfermeiro, determinar com precisão as características definidoras e os fatores relacionados que estão associadas ao DE Mobilidade Física Prejudicada, de forma que a exatidão do diagnóstico traçado esteja relacionada a uma boa qualidade no cuidado de enfermagem.

Desse modo, a relevância está em identificar a prevalência do DE Mobilidade Física Prejudicada e de seus componentes em pessoas com Esclerose Múltipla a contribuir para o estabelecimento de resultados positivos e intervenções de enfermagem eficazes para essa clientela. Além disso, o estabelecimento da razão de prevalência permite a inferência do DE da forma mais fidedigna, com maior acurácia, por estimar as chances de um indivíduo apresentar esse diagnóstico na presença de determinada característica e fator relacionado.

A partir das lacunas encontradas emergiu o seguinte questionamento: Qual a prevalência do diagnóstico de enfermagem Mobilidade Física Prejudicada em pessoas com Esclerose Múltipla? Existe associação entre esse diagnóstico, suas características definidoras e fatores relacionados? Quais as chances dessas pessoas terem esse DE na presença dessas características definidoras e fatores relacionados?

Assim, para responder ao questionamento o estudo teve como objetivos identificar a prevalência do diagnóstico de enfermagem mobilidade física prejudicada, suas características definidoras e fatores relacionados em pessoas com EM e verificar a associação entre ambos e suas razões de prevalência.

\section{REVISÃO DE LITERATURA}

As formas clínicas da EM apresentam-se em quatro tipos, a saber: forma Recorrente-Remitente (EMRR), forma Primariamente Progressiva (EMPP), forma Secundariamente Progressiva (EMSP) e forma Progressiva com Surtos (EMPS). A EMRR evolui em surtos que ocorrem de maneira súbita e podem deixar sequelas, é a forma mais comum da 
doença. Caracteriza-se por exacerbações seguidas por um grau variável de melhora do déficit neurológico, podendo ser completa ou evoluir com uma disfunção sintomática residual ${ }^{3}$.

A EMPP representa de $15 \%$ a $20 \%$ dos casos de EM como maior frequência em pessoas acima dos 40 anos e no sexo masculino. Embora não haja ocorrência de surtos, existe o acúmulo dos sintomas ao longo do tempo. A EMSP presente em 15 a $20 \%$ das formas de EM. Para o diagnóstico, é necessário realizar análise retrospectiva, uma vez que sua confirmação se baseia no prolongamento dos sintomas após seis meses. Inicialmente, o curso apresenta-se como a forma EMRR com posterior progressão, com ou sem surtos, discretas remissões e estabilizações ${ }^{4}$.

A EMPS é a mais rara dentre as formas clínicas, acometendo cerca de $5 \%$ ou menos dos pacientes. Apresenta início progressivo com presença posterior de surtos bem definidos e evolução progressiva ${ }^{3}$.

\section{METOdOLOGIA}

Trata-se de um estudo descritivo, transversal, com abordagem quantitativa realizada em um hospital universitário de referência para doenças neurológicas, no nordeste do Brasil.

A população do estudo constituiu-se de pacientes com diagnóstico médico confirmado de EM identificados e assistidos no referido hospital. Assim, calculou-se a amostra sem reposição, a partir do total de pacientes cadastrados no ambulatório de Neurologia do hospital, constituindo-se assim uma população de 158 pessoas. Para o cálculo do tamanho da amostra utilizou-se a fórmula para populações finitas, levando em consideração o nível de confiança de

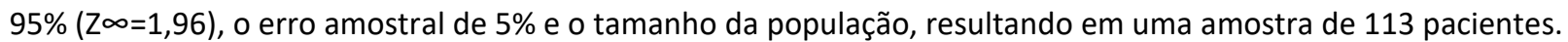

A amostra dos pacientes foi obtida através da amostragem por conveniência do tipo consecutiva. Para tanto, foram adotados os seguintes critérios de inclusão: ter sido diagnosticado clinicamente com EM, apresentar idade acima de 18 anos, estar cadastrado no ambulatório do hospital no período de coleta de dados. Foram critérios de exclusão: não estar em condições psíquicas e emocionais para participar do estudo. No intuito de verificar se o paciente apresentava condições físicas e psíquicas comprometidas, os pesquisadores se reportaram à equipe de enfermagem, consultas aos prontuários, assim como abordaram o paciente no sentido de realizar uma avaliação sobre seu estado geral de saúde, nível de consciência e orientação. O nível de consciência foi avaliado por meio do Miniexame de Estado Mental que se trata de uma medida dicotômica de 30 itens, com aplicação rápida e breve, que rastreia o comprometimento das funções cognitivas. Avalia orientação temporal e espacial, memória imediata e de evocação de palavras, cálculo, nomeação, repetição, execução de um comando, leitura, escrita e habilidade visomotora ${ }^{15}$.

Para verificar a condição psíquica dos pacientes com EM os pesquisadores previamente se reportavam ao prontuário, para analisar todo o histórico da doença e a evolução. Em seguida, reportavam-se à equipe de enfermagem para obter informações adicionais sobre o comportamento do paciente, sua orientação quanto a espaço e tempo.

Os dados foram coletados no período de março a setembro de 2017, por meio de um roteiro de entrevista e exame físico que contemplava os aspectos sociodemográficos, clínicos e comportamentais. O referido instrumento foi submetido à validação do conteúdo e aparência por dez docentes que desenvolvem estudos na área da Sistematização da Assistência de Enfermagem (SAE) e Neurologia, posteriormente as sugestões propostas foram contempladas no instrumento.

Logo em seguida foi realizado um treinamento teórico e prático para padronizar a coleta de dados com dois alunos de Iniciação Científica e três alunos de pós-graduação no nível de Mestrado, com carga horária de 12 horas semanais, desenvolvido por meio de aulas expositivas e dialogadas, além de discussões de casos clínicos com ênfase na abordagem a pessoas com EM. Após a etapa teórica do curso, realizou-se uma atividade prática de simulação de exame físico em pares, com o intuito de capacitar os pesquisadores e uniformizar a coleta de dados. Assim, após essa etapa, foi realizado sob a forma de pré-teste a aplicação do instrumento em dez pacientes com EM.

A elaboração dos diagnósticos foi processual, realizada simultaneamente com a coleta de dados, buscando identificar as características definidoras e os fatores relacionados/de risco de acordo com a NANDA-I, versão 2015-2017. Para a estruturação dos diagnósticos de enfermagem seguiram-se as etapas do julgamento clínico de Risner: Categorização dos dados, Identificação de lacunas de dados, Agrupamento dos dados relevantes em padrões, Comparação dos agrupamentos padrões normas e conceitos, Identificação com desvios e potencialidades em saúde, Proposições de relações etiológicas ${ }^{11}$.

Após essa etapa os resultados sofreram análise forma pareada a fim de assegurar um julgamento consensual e acurado. Em seguida, construiu-se um banco de dados utilizando o software Microsoft Excel 2009, registrando todas as variáveis dos instrumentos da pesquisa, como os respectivos diagnósticos de enfermagem, características definidoras e os fatores relacionados e os de risco identificados. 
Os dados foram analisados por meio da estatística descritiva e inferencial. Na análise descritiva, foram utilizadas as medidas de tendência central e de dispersão, sendo aplicado o teste de teste de Shapiro Wilk para verificar a distribuição da normalidade dos dados ${ }^{16}$. Também foram calculados a média e o desvio-padrão das variáveis quantitativas. Na análise inferencial, utilizaram-se o teste qui-quadrado de Pearson e teste exato de Fisher para verificar a associação dos diagnósticos de enfermagem com as características definidoras, e fatores relacionados, considerou-se o $p<0,05$, sendo calculadas também as razões de prevalência.

Diante disto, a pesquisa seguiu as normas nacionais e internacionais éticas em seres humanos, aprovada pelo Comitê de Ética em Pesquisa da Universidade Federal do Rio Grande do Norte (CEP/UFRN) sob o Parecer no 1.146.907.

\section{RESULTADOS}

Participaram do estudo 113 pacientes com idade mínima de 19 e máxima de 59 anos, sendo a média de idade de 51,16 anos $( \pm 12,6)$, do sexo masculino $(72,6 \%)$, solteiro $(66,4 \%)$, provenientes do interior do estado $(55,7 \%)$, sem filhos $(47,8 \%)$. As variáveis renda salarial e tempo de estudo apresentaram variação assimétrica, indicando que metade da amostra possuía renda de aproximadamente um salário mínimo e tinha até oito anos de estudo. O DE Mobilidade Física Prejudicada esteve presente em $89 \%$ dos pacientes.

A tabela 1 apresenta a prevalência do diagnóstico de enfermagem Mobilidade Física prejudicada, de suas características definidoras e fatores relacionados.

TABELA 1: Prevalência do diagnóstico de enfermagem Mobilidade Física prejudicada, de suas características definidoras e fatores relacionados em pessoas vivendo com EM. Natal/RN, 2017 ( $N=113)$

\begin{tabular}{|c|c|c|c|c|}
\hline \multirow[t]{2}{*}{ Variáveis } & \multicolumn{2}{|c|}{ Presença } & \multicolumn{2}{|c|}{ Ausência } \\
\hline & $\mathrm{n}$ & $\%$ & $\mathrm{n}$ & $\%$ \\
\hline \multicolumn{5}{|l|}{ Diagnóstico de Enfermagem } \\
\hline Mobilidade Física prejudicada & 78 & 89,0 & 35 & 31,0 \\
\hline \multicolumn{5}{|l|}{ Características definidoras } \\
\hline Alterações na marcha & 78 & 69,0 & 35 & 31,0 \\
\hline Instabilidade postural & 76 & 67,3 & 37 & 32,7 \\
\hline Movimentos descoordenados & 73 & 64,6 & 40 & 35,4 \\
\hline Redução nas habilidades motoras finas & 68 & 60,2 & 45 & 39,8 \\
\hline Redução nas habilidades motoras grossas & 65 & 57,5 & 48 & 42,5 \\
\hline Desconforto & 58 & 51,3 & 55 & 48,7 \\
\hline Dispneia ao esforço & 49 & 43,3 & 64 & 56,7 \\
\hline Redução na amplitude de movimentos & 44 & 38,9 & 70 & 61,1 \\
\hline Tremor induzido pelo movimento & 44 & 38,9 & 70 & 61,1 \\
\hline Movimentos lentos & 44 & 38,9 & 70 & 61,1 \\
\hline \multicolumn{5}{|l|}{ Fatores relacionados } \\
\hline Depressão & 70 & 61,1 & 43 & 38,1 \\
\hline Força muscular diminuída & 70 & 61,1 & 43 & 38,1 \\
\hline Prejuízo músculoesquelético & 68 & 60,2 & 45 & 39,8 \\
\hline Controle muscular diminuído & 68 & 60,2 & 45 & 39,8 \\
\hline Intolerância à atividade & 66 & 58,4 & 47 & 41,6 \\
\hline Prejuízos neuromusculares & 61 & 53,9 & 52 & 46,1 \\
\hline Massa muscular diminuída & 58 & 51,3 & 55 & 48,7 \\
\hline \multicolumn{5}{|l|}{ Diagnóstico de Enfermagem } \\
\hline Mobilidade Física prejudicada & 78 & 89,0 & 35 & 31,0 \\
\hline
\end{tabular}

Com base nos dados apresentados na Tabela 1, a característica definidora que mais se destacou, entre os pacientes que apresentaram o diagnóstico de enfermagem estudado foi alterações na marcha. Já entre os fatores relacionados os mais prevalentes foram depressão e força muscular diminuída. A Tabela 2 apresenta as características definidoras e fatores relacionados que apresentaram associação com o DE, bem como suas razões de prevalência nas pessoas com EM.

De acordo com a Tabela 2, cinco características definidoras e 3 fatores relacionados apresentaram associação estatisticamente significante quando relacionadas ao diagnóstico de enfermagem Mobilidade Física Prejudicada $(p<0,05)$, a saber: alterações na marcha; instabilidade postural; movimentos descoordenados; redução nas habilidades motoras finas e grossas; depressão; força muscular diminuída e prejuízo músculo esquelético. 
TABELA 2: Características definidoras e fatores relacionados que apresentaram associação com o diagnóstico de enfermagem Mobilidade Física prejudicada. Natal/RN, 2017 (N=113)

\begin{tabular}{|c|c|c|c|c|}
\hline \multirow{2}{*}{$\begin{array}{l}\text { Variáveis } \\
\text { Características Definidoras }\end{array}$} & \multicolumn{3}{|c|}{ DE Mobilidade Física prejudicada } & \multirow{2}{*}{ Estatísticas } \\
\hline & Presente & Ausente & Total & \\
\hline \multicolumn{5}{|l|}{ Alterações na marcha } \\
\hline Presente & 78 & 00 & 78 & $p=0,001^{1}$ \\
\hline Ausente & - & 35 & 35 & $\mathrm{RP}=3,582$ \\
\hline Total & 78 & 35 & 113 & IC95\%: 1,829 - 3,981 \\
\hline \multicolumn{5}{|l|}{ Instabilidade postural } \\
\hline Presente & 58 & 10 & 68 & $p=0,03^{2}$ \\
\hline Ausente & 20 & 25 & 45 & $\mathrm{RP}=2,671$ \\
\hline Total & 78 & 35 & 113 & IC95\%: 1,729 - 2,873 \\
\hline \multicolumn{5}{|l|}{ Movimentos descoordenados } \\
\hline Presente & 73 & 03 & 76 & $p=0,002^{1}$ \\
\hline Ausente & 05 & 32 & 37 & $\mathrm{RP}=1,351$ \\
\hline Total & 78 & 35 & 113 & IC95\%: 1,112 - 2,126 \\
\hline \multicolumn{5}{|c|}{ Redução nas habilidades motoras finas } \\
\hline Presente & 73 & 03 & 76 & $p=0,002^{1}$ \\
\hline Ausente & 05 & 32 & 37 & $\mathrm{RP}=1,351$ \\
\hline Total & 78 & 35 & 113 & IC95\%: 1,112 - 2,126 \\
\hline \multicolumn{5}{|c|}{ Redução nas habilidades motoras grossas } \\
\hline Presente & 73 & 03 & 76 & $p=0,002^{1}$ \\
\hline Ausente & 05 & 32 & 37 & $\mathrm{RP}=1,351$ \\
\hline Total & 78 & 35 & 113 & IC95\%: 1,112 - 2,126 \\
\hline \multicolumn{5}{|l|}{ Fatores relacionados } \\
\hline \multicolumn{5}{|l|}{ Depressão } \\
\hline Presente & 58 & 10 & 68 & $p=0,000^{*}$ \\
\hline Ausente & 20 & 25 & 45 & $\mathrm{RP}=1,450$ \\
\hline Total & 78 & 35 & 113 & IC95\%: 0,989 - 2,723 \\
\hline \multicolumn{5}{|l|}{ Força muscular diminuída } \\
\hline Presente & 48 & 22 & 70 & $p=0,038 * *$ \\
\hline Ausente & 30 & 13 & 43 & $\mathrm{RP}=1,684$ \\
\hline Total & 78 & 35 & 113 & IC95\%: 0,889 - 2,545 \\
\hline \multicolumn{5}{|l|}{ Prejuízo músculo esquelético. } \\
\hline Presente & 48 & 22 & 70 & $\mathrm{p}=0,038^{*} *$ \\
\hline Ausente & 30 & 13 & 43 & $\mathrm{RP}=1,684$ \\
\hline Total & 78 & 35 & 113 & IC95\%: 0,889 - 2,545 \\
\hline
\end{tabular}

${ }^{1}$ Teste exato de Fisher; ${ }^{2}$ Teste qui-quadrado de Pearson; $p<0,05 ; \mathrm{RP}=$ Razão de prevalência; IC = Intervalo de confiança de $95 \%$.

Em relação às razões de prevalência das características anteriormente citadas, as chances das pessoas com EM desenvolverem o diagnóstico de enfermagem Mobilidade Física Prejudicada na presença dessas características foram de aproximadamente: 3,5 vezes para alterações na marcha, 2,6 vezes para instabilidade postural, 1,3 vezes para movimentos descoordenados, 1,3 vezes para redução nas habilidades motoras finas e 1,3 vezes para redução nas habilidades motoras, quando comparados aos pacientes que não apresentaram essas características e fatores.

Ademais, apesar da associação estatisticamente significante entre os fatores relacionados; depressão; força muscular diminuída e prejuízo músculo esquelético com o DE Mobilidade Física Prejudicada, não há como estabelecer a magnitude da associação, uma vez que a razão de prevalência não apresentou significância estatística devido a presença do valor 1 no intervalo de confiança.

\section{DISCUSSÃO}

Os estudos de prevalência de DE asseveram uma contribuição positiva para a prática do enfermeiro na assistência, facilitando o planejamento das ações de enfermagem prioritárias para uma determinada população. Deste modo, é afirmada a Enfermagem enquanto ciência, por utilizar um sistema de classificação próprio proposto pela NANDA-I, como referencial teórico próprio da enfermagem, colaborando para as práticas assistenciais entre os enfermeiros ${ }^{17}$.

Neste contexto, a tomada de decisão do enfermeiro é caracterizada pela sua avaliação minuciosa e rigorosa de evidências relevantes sobre um problema de enfermagem, iniciando pela síntese de dados expressivos e pela obtenção 
de informações sobre possíveis intervenções, tratamentos e meios de controle e manutenção, levando em consideração a expertise do profissional e a vontade do paciente ${ }^{18}$.

Ao elencar o DE Mobilidade Física Prejudicada da NANDA-I, o enfermeiro simultaneamente planejará o seu plano de cuidado baseado no diagnóstico delimitado, através da Classificação dos Resultados de Enfermagem (NOC) ${ }^{19}$, avaliando os seus indicadores de forma a programar as possíveis intervenções, utilizando-se da Classificação das Intervenções de Enfermagem (NIC) ${ }^{20}$, e dessa forma, sistematizando a assistência e o cuidado de enfermagem.

$\mathrm{Na}$ EM a perda de mielina causada pela formação de placas desmielinizantes no sistema nervoso central (SNC), leva a um comprometimento da barreira hematoencefálica, inflamação, desmielinização, remielinização, perda e depressão de oligodendrócitos. Tais características sucedem devido a uma combinação de diversos fatores ambientais e predisposição genética individual que conduzem ao aparecimento de clones de linfócitos T autorreativos na corrente sanguínea e uma desregulação dos processos reguladores imunológicos no sangue periférico e localmente no SNC, atingindo áreas como substância branca periventricular e subcortical, corpo caloso, córtex, estrutura da fossa superior, nervos ópticos e medula²1.

Portanto, o dano às fibras nervosas causado pela desmielinização, ocorre devido à interrupção dos impulsos nervosos na condução nervosa prejudicada. Essa interrupção causa fadiga e fraqueza muscular, onde os músculos não recebem os impulsos nervosos de que necessitam para trabalhar de forma eficaz, o que pode resultar em redução da resistência ${ }^{22}$.

Esse quadro apresentado pela EM, vai implicar na limitação no movimento físico independente e voluntário do corpo ou das suas extremidades, que consequentemente vai interferir de forma significativa na mobilidade de pacientes acometidos por EM, dessa forma, faz-se presente e prevalente o DE Mobilidade Física Prejudicada em grande parte dos casos de pessoas com o diagnóstico de EM.

Diante do DE Mobilidade Física Prejudicada, o principal resultado de enfermagem classificado é a mobilidade, com os indicadores avaliados como tipo de marcha, coordenação e movimento muscular ${ }^{19}$.

As lesões desmielinizantes no cerebelo e tratos cerebelares são comuns na EM, e caracterizam-se por tremores posturais ou intencionais aos movimentos voluntários, que quando exacerbados levam a incapacidades nas atividades da vida diária e fraqueza da musculatura do tronco. Diante disso, o enfermeiro pode implementar atividades de extrema importância que auxiliem na instabilidade postural, no tônus muscular e fadiga.

Dentre essas atividades pode-se citar o auxílio e orientação de exercícios de transferências de objetos de um lado do corpo para o outro, pois ajuda a manter o tronco e a estabilidade das articulações proximais durante a atividade motora; a avaliação conjunta ao fisioterapeuta para melhor definição na realização dos exercícios conforme apropriado; oferecimento de informações sobre a função dos músculos, a fisiologia dos exercícios e as consequências do desuso; oferta de um ambiente repousante ao paciente após períodos de exercícios, dessa maneira, avaliando os indicadores do resultado de enfermagem mobilidade ${ }^{23,24}$.

Dentre as lesões decorrentes do processo de desmielinização, a redução das habilidades motoras grossas e finas pode aparecer no curso da EM, para isso é importante que o enfermeiro adote atividades juntamente com o profissional fisioterapeuta que auxiliem o paciente na realização das atividades de vida diária afetada como auxiliar nas atividades de coordenação para mão e membro superior, trabalhando a garra e a pinça, preensão, palpando objetos de tamanhos e espessuras variáveis, realizar desenhos sobre diagramas, atentando para desenvolver atividades com sequências progressivas, auxiliar em atividades de deambulação, subida de degraus e posicionamento do corpo, conforme apropriado ${ }^{19,20}$

As alterações na marcha, os movimentos descoordenados e a redução na amplitude de movimentos também estão relacionados às lesões desmielinizantes no cerebelo, tais lesões podem ser causa para fraqueza, espasticidade, alteração sensorial ou ataxia, bem como a combinação desses fatores ${ }^{25}$.

O tratamento de reabilitação para pessoas que vivem com EM geralmente inclui treinamento cardiovascular, de força e equilíbrio. No entanto, devido à variável apresentação na EM e à ampla gama de sintomas, nenhuma abordagem de intervenção foi identificada como padrão-ouro. Portanto, o tratamento é tipicamente individualizado, dependendo dos sinais e sintomas individuais do paciente. Entre as muitas apresentações comuns em pessoas com esclerose múltipla estão as queixas de tontura, instabilidade e falta de equilíbrio. Embora esses sintomas possam ter contribuições do desempenho muscular alterado, as disfunções do sistema vestibular são uma possibilidade real e geralmente são negligenciadas ${ }^{25}$.

A partir disso, o enfermeiro implementará atividades voltadas ao auxílio e orientação no uso de dispositivos que auxiliam na locomoção, barras de apoio, uso de órteses, orientar, demonstrar e auxiliar na deambulação quando possível. Ao final das atividades, analisar os avanços dos indicadores do resultado de enfermagem sugerido ${ }^{26}$. 
Nesse contexto de implementação de atividades, o enfermeiro deve levar em conta o papel interdisciplinar para que ocorram interação e participação do paciente no manuseio, facilitação, e na ênfase na qualidade do movimento, refletindo assim no alto desempenho e no papel ativo, o que enriquece os processos de raciocínio e tomada de decisão ${ }^{26}$.

Contudo, o tremor induzido pelo movimento não está facilmente associado a um sítio neuroanatômico, uma vez que a EM é uma doença multifocal. A predominância desses tremores sugere lesões no cerebelo que apresenta sinais e sintomas como paresias, espasticidades, reflexos exagerados, espasmos flexores e extensores involuntários ${ }^{27}$.

$O$ tremor em pessoas com EM é um sintoma frequente e debilitante, com uma fisiopatologia relativamente pouco compreendida e contribui muito para a incapacidade desses pacientes. Esse tremor raramente se apresenta com o componente de ação de alta frequência observado nos distúrbios do tremor primário; pelo contrário, é a combinação de várias características, particularmente as cerebelares. Portanto, os medicamentos raramente são eficazes, enquanto a cirurgia pode fornecer resultados variáveis, dependendo da gravidade da ataxia subjacente ${ }^{27}$.

Nesse caso, o enfermeiro pode desenvolver atividades como orientar o paciente para evitar movimentos rápidos, forçados ou pendulares para prevenir a estimulação exagerada ao reflexo miotático, sempre associando a mensuração dos indicadores do resultado de enfermagem mobilidade.

Além da deficiência nos músculos do tronco e membros, os músculos respiratórios também são afetados, causando uma diminuição na força e resistência. A fraqueza da musculatura respiratória, incluindo o diafragma e músculos da parede torácica, resulta em tosse ineficiente, aumentando as chances de infecção, e respiração superficial, levando à hipoventilação e, consequentemente, à hipoxemia e hipercapnia. Com a hipoventilação, as bases pulmonares são pouco ventiladas e os pulmões e as paredes do tórax tornam-se menos elásticos resultando em complicações respiratórias ${ }^{28}$.

Com isso, a dispneia ao esforço se faz presente em indivíduos com EM, por apresentarem menor tolerância a exercícios físicos em decorrência da redução da força muscular. Além disso, essas anomalias também podem aumentar o risco de hospitalizações e morbidades frequentes e podem reduzir a expectativa de vida. Infelizmente, o impacto da intervenção do exercício nessas anomalias é desconhecido. Portanto, sugere-se que o indivíduo com EM seja rastreado sistematicamente quanto à função muscular, cardíaca, ventilatória e metabólica ${ }^{29}$

Para reverter ou atenuar esse quadro, o enfermeiro desenvolverá atividades a fim de minimizar danos e melhorar taxas de fluxo e volumes pulmonares, como posicionar o paciente visando ao alívio da dispneia, elevar a cabeceira da cama, auscultar sons pulmonares, observando áreas de ventilação diminuída ou ausente, além da presença de ruídos adventícios, monitora a ocorrência de fadiga nos músculos respiratórios, monitorar frequência, ritmo, profundidade e esforço nas respirações ${ }^{30}$.

Podem ocorrer também, complicações secundárias na EM que dificultam a reabilitação dos pacientes, como alterações psicossociais. Estas alterações implicam numa reestruturação e adaptação, uma vez que estas pessoas têm que aprender a gerir a nova situação de doença. Deste modo, a vivência da doença crônica depende das características individuais, das crenças culturais, da aceitação e do que se espera da vida, tendo forte impacto nos projetos futuros, na educação, relações sociais, casamento, vida familiar e profissional ${ }^{31}$.

Ainda em relação às alterações psicossociais, a depressão é um fator relacionado à EM. Estudo português desenvolvido com pessoas com EM aponta que a diminuição dos contatos sociais e a perda de interesse em atividades antes consideradas prazerosas podem ser resultado do diagnóstico e das consequências físicas que acompanham a doença, ademais, estes dois aspetos poderão conduzir a maior sintomatologia depressiva que, por sua vez, irá refletir em maior escala no isolamento dos indivíduos ${ }^{32}$

Diante disso, é de suma relevância que o enfermeiro programe atividades que auxiliem no autocuidado dos pacientes, a fim de melhorar o enfrentamento e a percepção da imagem corporal do mesmo, como encorajar o paciente a realizar as atividades normais da vida diária como conforme seu nível de capacidade, encorajar na independência, mas interferir quando o paciente tiver dificuldades no desempenho e dar assistência até que o paciente esteja completamente capacitado a assumir o autocuidado 20

Sendo assim, é importante que indivíduo e família necessitem de acompanhamento por parte dos profissionais de saúde, no sentido de serem minimizados os fatores de risco que ponham em causa a singularidade do indivíduo e a dinâmica familiar. O apoio passa pelo esclarecimento e transmissão de informação de qualidade sobre a natureza e evolução da doença, gestão de sinais e sintomas, identificação de sinais de desgaste físico e emocional, promovendo a aquisição de estratégias de enfrentamento e de adaptação, melhorando a qualidade de vida destas pessoas ${ }^{31,33}$.

Por fim, compreende-se que ao indicar o DE Mobilidade Física Prejudicada em pessoas com EM o enfermeiro tem em suas mãos a utilização de uma linguagem única, que padroniza sua documentação e possibilita comparações e 
avaliação da efetividade de suas intervenções na resolução de problemas de saúde dos indivíduos, favorecendo sua coleta de dados e ações prioritárias, subsidiando seu método de trabalho ${ }^{34}$.

\section{CONCLUSÃO}

O diagnóstico Mobilidade Física Prejudicada apresentou-se com elevada frequência na amostra investigada. As características definidoras que apresentaram associação estatística com o referido diagnóstico foram: alterações na marcha; instabilidade postural; movimentos descoordenados; redução nas habilidades motoras finas e grossas. Já os fatores relacionados que apresentaram associação com o diagnóstico foram: depressão; força muscular diminuída e prejuízo músculo esquelético. Dentre estes, alterações na marcha e instabilidade postural apresentaram as maiores chances para o desenvolvimento desse diagnóstico.

Diante o exposto, compete ao enfermeiro identificar as necessidades de intervenções que dizem respeito à capacidade funcional do indivíduo para realização das suas atividades diárias, assim como, fazer com que o portador da EM seja produtivo e atinja um bom nível de resistência, promovendo mobilidade, acessibilidade e participação quanto ao seu autocuidado, potencializando seu rendimento funcional e pessoal, através do planejamento do cuidado e da assistência qualificada por meio do processo de enfermagem.

Dentre as limitações do estudo, está o fato de a avaliação clínica ser um processo subjetivo, diante disso, o processo diagnóstico está sujeito a incertezas, trazendo implicações para os resultados esperados e intervenções específicas de enfermagem. Ademais, a concretização e divulgação deste e de outros estudos relacionados ao tema abordado podem subsidiar os profissionais de enfermagem quanto à elaboração e implementação de uma prática de enfermagem científica, humana e holística. Assim como, trazer reflexões a respeito do estudo promovendo novas pesquisas.

\section{REFERÊNCIAS}

1. Marques VD, Passos GR, Mendes MF, Callegaro D, Lana-Peixoto MA, Comini-Frota ER, et al. Brazilian Consensus for the Treatment of Multiple Sclerosis: Brazilian Academy of Neurology and Brazilian Committee on Treatment and Research in Multiple Sclerosis. Arq. Neuropsiquiatr. [Internet]. 2018 [cited 2018 Jun 02]; 76(8): 539-554. DOI: http://dx.doi.org/10.1590/0004-282x20180078.

2. Ministério da Saúde (Br). Pacientes terão novo medicamento. Brasília (DF): Ministério da Saúde; 2017 [cited 2018 Jun 02]. Available from: http://portalms.saude.gov.br/noticias/agencia-saude/28579-pacientes-terao-novo-medicamento.

3. Associação Brasileira de Esclerose Múltipla - ABEM [site de internet]. O que é Esclerose Múltipla? [cited 2018 Jun 02] Available from: http://abem.org.br/esclerose/o-que-e-esclerose-multipla/.

4. Recomendações Esclerose Múltipla [site de internet]. [cited 2018 Jun 02]. Available from: http://formsus.datasus.gov.br/novoimgarq/14491/2240628_109700.pdf.

5. Huang W, Chen W, Zhang X. Multiple sclerosis: Pathology, diagnosis and treatments. Exp Ther Med. [Internet]. 2017 [cited 2018 Jun 02]; 13 (6): 3163-66. DOI: http://dx.doi.org/10.3892/etm.2017.4410

6. Magyari M. Gender differences in multiple sclerosis epidemiology and treatment response. Dan Med J. [Internet]. 2016 [cited 2018 Jun 02]; 63 (3): B5212. Available from: https://ugeskriftet.dk/files/scientific_article_files/2018-11/b5212.pdf

7. Ribas MLV, Ribeiro NMS. Analysis of fatigue in patients with multiple sclerosis: a preliminary study. Cad. Pós-Grad. Distúrb. Desenvolv. [Internet]. 2017 [cited 2018 Jun 02]; 17 (1): 77-86. Available from: http://pepsic.bvsalud.org/scielo.php?script=sci_abstract\&pid=S1519-03072017000100009\&lng=pt\&nrm=iso\&tlng=en

8. Baixinho CL, Mertens J, Duarte AF, Teixeira FM, Quental IA, Martins SS, et al. Nursing interventions promoting functionality among adults with multiple sclerosis: integrative review. Rev. enferm. UFPE on line. [Internet]. 2016 [cited 2018 Jun 02]; 10 (Supl. 2): 838-47. Available from: https://periodicos.ufpe.br/revistas/revistaenfermagem/article/view/11027

9. Yhang B, Sampaio HAC, Almeida JAC, Melo MLP. Nutrition in Multiple Sclerosis: An integrative review of scientific publications from the last 5 years. Rev. chil. nutr. [Internet]. 2019 [cited 2019 Oct 12]; 46 (3): 230-38. Available from: https://scielo.conicyt.cl/pdf/rchnut/v46n3/0717-7518-rchnut-46-03-0230.pdf

10. Cavenaghi VB, Dobrianskyj FM, Olival GS, Carneiro RPCD, Tilbery CP. Characterization of the first symptoms of multiple sclerosis in a Brazilian center: cross-sectional study. Sao Paulo Med. J. [Internet]. 2017 [cited 2019 Nov 11]; 135 (3): 222-5. Available from: http://www.scielo.br/pdf/spmj/v135n3/1806-9460-spmj-135-03-00222.pdf

11. Ministério da Saúde (Br) [Homepage]. Medicamento para esclerose múltipla dará maior qualidade de vida aos pacientes. Brasília: Ministério da Saúde; 2019 [cited 2019 Mar 20]. Available from: http://portalms.saude.gov.br/noticias/agenciasaude/45084-medicamento-para-esclerose-multipla-no-sus-dara-maior-qualidade-de-vida-aos-pacientes.

12. Costa TM, Domingos MM, Silva BC, Neto VLS, Negreiros RV, Silva RA. Diagnostics, results and nursing interventions in patients with multiple sclerosis. Rev. cuba enferm. [Internet]. 2017 [cited 2018 Jun 02]; 33 (3): 1-6. Available from: http://www.revenfermeria.sld.cu/index.php/enf/article/view/1331.

13. Herdman TH, Kamitsuru S. Diagnósticos de enfermagem da Nanda: definições e classificação 2015-2017. 10a ed. Porto Alegre (RS): Artmed; 2015.

14. Ozkul C, Guclu-Gunduz A, Irkec C, Fidan I, Aydin Y, Ozkan T, et al. Effect of combined exercise training on serum brain-derived neurotrophic factor, suppressors of cytokine signaling 1 and 3 in patients with multiple sclerosis. J Neuroimmunol [Internet]. 2018 [cited 2018 Jun 02]; 15; 316: 121-9. DOI: https://doi.org/10.1016/j.jneuroim.2018.01.002. 
15. Melo DM, Barbosa AJG, Neri AL. Minimental State Examination: Validity evidence based on internal structure. Aval. psicol. [Internet]. 2017 [cited 2019 Nov 06]; 16 (2): 161-8. Available from: http://dx.doi.org/10.15689/AP.2017.1602.06

16. Santos GAB, Couto NF, Almeida SA, Silva CCRD, Fernandes Filho J, Silva S. Comparison and Agreement of body fat in children among 3 doubly indirect methods. Rev. Fac. Med. [Internet]. 2017 [cited 2018 Jun 02]; 65 (4): 609-14. DOI: http://dx.doi.org/10.15446/revfacmed.v65n4.60315.

17. Bittencourt GKGD, Crossetti MGO. Habilidades de pensamento crítico no processo diagnóstico de enfermagem. Rev Esc Enferm USP [Internet]. 2013 [cited 2019 Mar 20]; 47(2): 341-7. Available from: http://www.scielo.br/pdf/reeusp/v47n2/10.pdf.

18. Moura LA, Araújo JNM, Pitombeira DO, et al. Risk factors of the nursing diagnosis in the safety/protection domain: integrative review. Cogitare Enferm. [Internet]. 2016 [cited 2019 Mar 20]; 21(4): 01-8. DOI: http://dx.doi.org/10.5380/ce.v21i4.45145

19. Moorhead S, Johnson M, Maas ML, Swanson E. Classificação dos resultados de enfermagem (NOC). 5a ed. Rio de Janeiro: Elsevier; 2016.

20. Bulechek GM, Butcher HK, Dochterman JM, Wagner CM. Classificação das intervenções de enfermagem (NIC). 6a ed. Rio de Janeiro: Elsevier; 2016.

21. Corallo F, Bonanno L, Di Cara M, Rifici C, Sessa E, D'Aleo G, et al. Therapeutic adherence and coping strategies in patients with multiple sclerosis: an observational study. Medicine [Internet]. 2019 [cited 2019 Mar 20]; 98 (29): e16532. DOI: https://doi.org/10.1097/MD.0000000000016532

22. Faissner S, Gold R. New therapeutic approaches in progressive multiple sclerosis. Fortschr Neurol. Psychiatr. [Internet] 2019 [cited 2019 Mar 20]; 87 (11): 653-71. DOI: http://dx.doi.org/10.1055/a-0880-0073

23. Oliveira-Kumakura ARS, Bezutti LM, Silva JLG, Gasparino RC. Functional and self-care capacity of people with multiple sclerosis. Rev. Latino-Am. Enfermagem [Internet]. 2019 [cited 2019 Nov 11]; 27: e3183. DOI: http://dx.doi.org/10.1590/15188345.3068 .3183

24. Costa TMS, Souza Neto VL, Domingos MMC, Silva BCO, Rodrigues IDCV, Silva RAR. A profile of nursing diagnoses in patients with multiple sclerosis: a cross-sectional study. Online Braz. J. Nurs. [Internet]. 2016 [cited 2019 Nov 11]; 15 (3): 433-42. DOI: http://dx.doi.org/10.17665/1676-4285.20165383

25. Loyd BJ, Fangman A, Peterson DS, Gappmaier E, Schubert MC, Thackery A, et al. Rehabilitation to improve gaze and postural stability in people with multiple sclerosis: study protocol for a prospective randomized clinical trial. BMC Neurol. [Internet]. 2019 [cited 2019 Nov 11]; 19: 119. DOI: https://doi.org/10.1186/s12883-019-1353-z

26. Lahelle AF, $\varnothing$ berg GK, Normann B. A group-based, individualized physiotherapy intervention for people with multiple sclerosis: a qualitative study. Physiother Res Int. [Internet]. 2018 [cited 2019 Nov 11]; 23 (4): e1734 DOI: https://doi.org/10.1002/pri.1734

27. Soh D, Fasano A. Multiple sclerosis tremor: are technical advances enough? Lancet Neurol. [Internet]. 2017 [cited 2019 Nov 11]; 16 (9): 678-9. DOI: https://doi.org/10.1016/S1474-4422(17)30198-9

28. Copetti PR, Rodrigues LS, Pissolato JS, Brondani SC, Fagúndez MRP, Filippin NT. Respiratory muscle training in multiple sclerosis subjects: a systematic review. Biomotriz [Internet]. 2014 [cited 2019 Mar 22]; 8 (2): 61-76. Available from: http://revistaeletronica.unicruz.edu.br/index.php/BIOMOTRIZ/article/view/1891.

29. Wens I, Eijnde BO, Hansen D. Muscular, cardiac, ventilatory and metabolic dysfunction in patients with multiple sclerosis: Implications for screening, clinical care and endurance and resistance exercise therapy, a scoping review. J. Neurol. Sci. [Internet]. 2016 [cited 2019 Mar 22]; 15; 367: 107-21. DOI: https://doi.org/10.1016/j.jns.2016.05.050.

30. Goodwin E, Hawton A, Green C. Using the Fatigue Severity Scale to inform healthcare decision-making in multiple sclerosis: mapping to three quality-adjusted life-year measures (EQ-5D-3L, SF-6D, MSIS-8D). Health Qual. Life Outcomes. [Internet]. 2019 [cited 2019 Mar 22]; 17 (1): 136. DOI: https://doi.org/10.1186/s12955-019-1205-y.

31. Neves CFS, Rente JAPS, Ferreira ACS, Garrett ACM. Quality of life of people with multiple sclerosis and their caregivers. Referência [Internet]. 2017 [cited 2019 Mar 22]; IV: 12. DOI: https://doi.org/10.12707/RIV16081

32. Pinto CR, Guerra M. The influence of meaning in life and depression in multiple sclerosis. Anál. psicol. [Internet]. 2018 [cited 2019 Mar 22]; 36 (4): 439-53. DOI: https://dx.doi.org/10.14417/ap.1394

33. Lopera-Vásquez JP. Flaws in the doctor-patient relationship in multiple sclerosis. Physis [Internet]. 2018 [cited 2019 Nov 12]; 28 (3): e280315. DOI: http://dx.doi.org/10.1590/S0103-73312018280315

34. Hirano GSB, Lopes CT, Barros ALBL. Development of research on nursing diagnoses in Brazilian graduate programs. Rev. Bras. Enferm. [Internet]. 2019 [cited 2019 Oct 12]; 72 (4): 926-32. Available from: http://www.scielo.br/pdf/reben/v72n4/00347167-reben-72-04-0926.pdf 